\title{
Performance Tradeoff with Routing Protocols for Radio Models in Wireless Sensor Networks
}

\author{
Manju Bala, Lalit Awasthi
}

Department of Computer Science and Engineering, National Institute of Technology, Hamirpur, India.

Email: sharmamanjub@rediffmail.com

Received November $16^{\text {th }}, 2010$; revised February $15^{\text {th }}, 2011$; accepted February $18^{\text {th }}, 2011$.

\begin{abstract}
In this paper, we have simulated and evaluated the performance tradeoff with routing protocols: Constrained Flooding, the Real-Time Search and the Adaptive Tree on MICA and MICAz platform with different radio models using PROWLER for wireless sensor networks. The simulation results establish that the MICAz motes give low latency, high throughput, high energy consumption, low efficiency but better lifetime while the MICA motes give high success rate and less loss rate. It has been, thus, concluded that in case of all the radio models the MICAz is preferably better than MICA in applications where energy is a constraint. Moreover, use of MICAz motes increases the network lifetime in comparison to MICA for the radio models. Further, the AT protocol can be applied to achieve better energy consumption, efficiency and lifetime in real time for wireless sensor networks.
\end{abstract}

Keywords: Constraint-Based Routing, Meta-Strategies, Real-Time Reinforcement Learning, Routing Tree, Wireless Sensor Networks

\section{Introduction}

Wireless sensor networks (WSNs) contain hundreds or thousands of sensor nodes equipped with sensing, computing and communication abilities. Each node has the ability to sense elements of its environment, perform simple computations, and communicate among its peers or directly to an external base station (BS) [1]. Routing in sensor network, however, has very different characteristics than routing in traditional communication networks. First of all, address-based destination specification is replaced by a more general feature-based specification, such as geographic location [2] or information gain [3]. Secondly, routing metrics are not just shortest Latency, but usually multiple objectives, including energy usage [4] and information density [3]. Thirdly, in addition to peer-to-peer communication, multicast (one-to-many) and converge-cast (many-to-one) are major traffic patterns in sensor networks. Even for peer-to-peer communication, the source/destination pairs often are dynamic (changing from time to time) or mobile (moving during routing).

Lot of research has been done recently on routing mechanisms that take QoS and trade-off specifications into consideration. Message-initiated Constrained-Based Routing (MCBR) [5] for wireless ad-hoc sensor networks is one of them. MCBR is a framework of routing mechanisms composed of the explicit specification of constrained-based destinations, route constraints and QoS requirements for messages, and a set of QoS-aware meta-strategies.

Three distributed Meta routing strategies based on real-time reinforcement learning [6]: Real-Time Search (RTS) [7], Constrained Flooding (CF) [8], and Adaptive Tree (AT) [9] have also been proposed. All of these use the same reinforcement learning core, which estimates and updates the cost from the current node to the destination. In contrast to most existing QoS routing, learning-based meta-strategies do not create and maintain explicit routes; instead, packets discover and improve the routes over time.

However, it has been observed in the literature that the performance evaluation of the routing protocols for wireless sensor networks has not been carried out in the presence of realistic radio models on different mote platforms. Thus the main contribution of this paper is performance trade-off with routing protocols CF, RTS and AT for wireless sensor networks under the influence of various realistic fading models in a simulated environment for MICA and MICAz on MATLAB platform. The trade-off comparison has been done on the basis of various performance metrics latency $(\mathrm{sec})$, throughput (data 
packets/sec), loss rate, success rate, energy consumption, energy efficiency and lifetime (years). Here the performance evaluation is done by using the event-driven simulator PROWLER integrated with Routing Modeling Application Simulation Environment (RMASE) [10].

The organization of the paper is as follows: after brief introduction in Section 1, the Section 2 describes the simulation model under different radio models. Section 3 reported the comparative findings for CF, RTS \& AT protocols with the influence of Normal Radio Model (NRM), Radio Model with SINR (RMSINR); Radio Model with Rayleigh Fading (RMRYF); and Radio Model with Rician Fading (RMRCF) for targeted Berkeley MICA and MICAz motes. Finally Section 4 concludes the paper.

\section{Simulation Model}

Currently there are many network simulators are available such as SensorSim [11], TOSSIM [12], NS2 [13], OPNET [14]. In this paper a matlab-based simulator PROWLER has been used which was developed by NEST, Vanderbilt University [15]. It provides an easy way of application prototyping with nice visualization capabilities. It is an event-driven tool that simulates the nondeterministic nature of the communication channel and the low-level communication protocol of the wireless sensor nodes [16]. To produce replicable results while testing the application, prowler can be set to operate in deterministic mode also. It can incorporate arbitrary number of nodes on arbitrary and even dynamic topology. Prowler models all the important aspects of the communication channel and the application. Here the following radio and MAC models have been compared to investigate their performance.

\subsection{Radio, MAC and Routing Models}

The simple radio model in PROWLER attempts to simulate the probabilistic nature in wireless sensor communication observed by many. The propagation model determines the strength of a transmitted signal at a particular point of the space for all transmitters in the system. Based on this information the signal reception conditions for the receivers can be evaluated and collisions can be detected. Here the work reported in [15] has been extended by developing a new radio model Rician. Subsequently the comparative findings have been reported with different routing protocols CF, RTS and AT for wireless sensor networks in a simulated environment for MICA and MICAz Berkley motes.

The transmission model is given by [17]:

$$
P_{\text {rec, ideal }}(d) \leftarrow P_{\text {transmit }}\left(1 /\left(1+d^{\gamma}\right)\right) \text {, where } 2 \leq \gamma \leq 4
$$

$$
P_{\text {rec }}(i, j) \leftarrow P_{\text {rec }, \text { ideal }}\left(d_{i, j}\right)(1+\alpha(i, j))(1+\beta(t))
$$

where $P_{\text {transmit }}$ is the signal strength at the transmitter and $P_{\text {rec, ideal }}(d)$ is the ideal received signal strength at distance $d, \alpha$ and $\beta$ are random variables with normal distributions $N\left(0, \sigma_{\alpha}\right)$ and $N\left(0, \sigma_{\beta}\right)$, respectively.

A network is asymmetric if $\sigma_{\alpha}>0$ or $\sigma_{\beta}>0$. In Equation (2), $\alpha$ is static depending on locations $i$ and $j$ only, and $\beta$ is dynamic which changes over time. A node $j$ can receive a packet from node $i$ if $P_{\text {rec }}(i, j)>\Delta$ where $\Delta>0$ is the threshold. There is a collision if two transmissions overlap in time and both could be received successfully. Furthermore, an additional parameter $p_{\text {error }}$ models the probability of a transmission error caused for any other reason. The default radio model in PROWLER has $\gamma=2$, $\sigma_{\alpha}=0.45, \sigma_{\beta}=0.02, \Delta=0.1$ and $p_{\text {error }}=0.05$. Figure 1(a) shows a snapshot of the radio reception curves in this model.

The transmission model for radio model with SINR in PROWLER is given by:

$$
P_{\text {rec }}(i, j) \leftarrow P_{\text {rec }, \text { ideal }}\left(d_{i, j}\right)(1+\alpha(i, j))
$$

where all the variables have the same values and meaning as in case of normal radio model with SINR described above. Figure 1(b) shows a snapshot of the radio reception curves in this model.

The transmission model for radio model with Rayleigh fading in PROWLER is given by:

$$
P_{r e c}(i, j) \leftarrow P_{r e c, \text { ideal }}\left(d_{i, j}\right)(R)
$$

where $R$ is a random variable with exponential distribution $(\mu=1)$. The coherence time is $\tau=1 \mathrm{sec}$. Figure 1(c) shows a snapshot of the radio reception curves in this model.

The transmission model for radio model with Rician fading in PROWLER is given by:

$$
P_{\text {rec }}(i, j) \leftarrow \text { filter }\left(\text { chan, } P_{\text {rec, }, \text { ideal }}\left(d_{i, j}\right)\right)
$$

where chan $=$ Ricianchan $(t s, f d, k)$. Here $t s=1^{-4} \mathrm{sec}$ is the sampling time, $f d=100 \mathrm{kHz}$ is the Doppler shift and $k=5$ is the Rician factor. Figure 1(d) shows a snapshot of the radio reception curves in this model.

The MAC layer communication is modeled by a simplified event channel that simulates the Berkeley motes' [18] CSMA MAC protocol. When the application emits the Send Packet command, after a random Waiting Time interval the MAC layer checks if the channel is idle. If not, it continues the idle checking until the channel is found idle. The time between idle checks is a random interval characterized by Backoff Time. When the channel is idle the transmission begins, and after Transmission Time the application receives the Packet Sent event. 


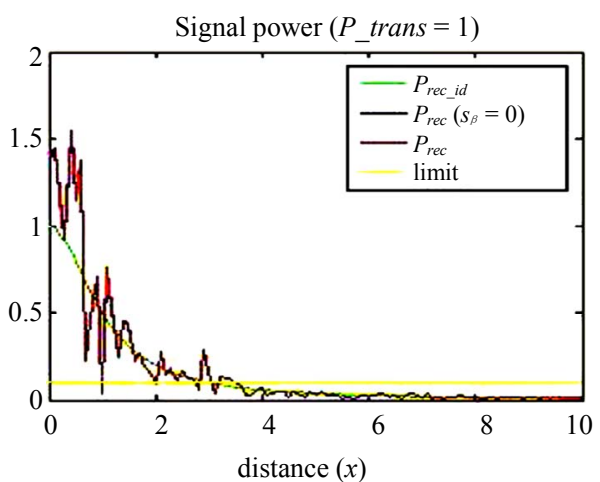

(a)

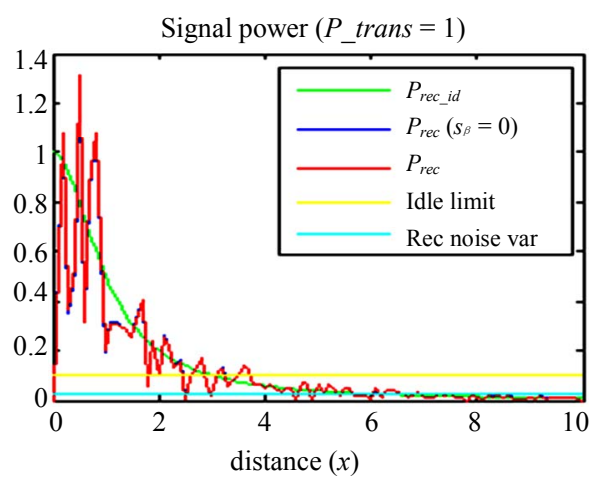

(b)

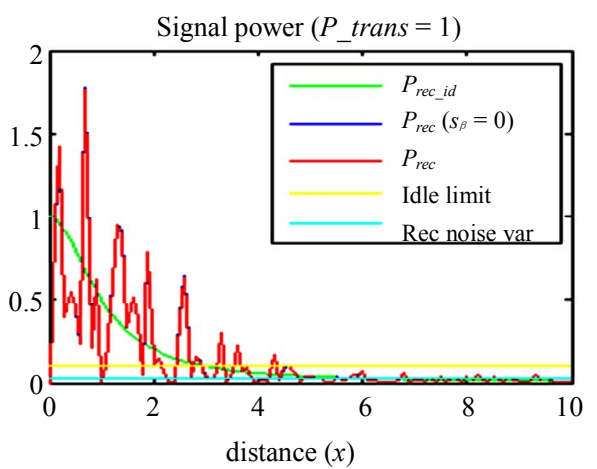

(c)

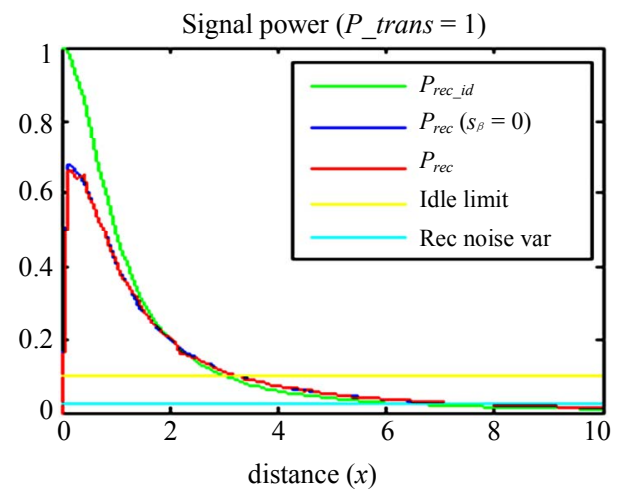

(d)

Figure 1. Radio reception curves for (a) NRM (b) RMSINR (c) RMRYF (d) RMRCF.
After the reception of a packet on the receiver's side, the application receives a Packet Received or Collided Packet Received event, depending on the success of the transmission. An application built on PROWLER integrated with RMASE which provides network generation and performance evaluations for routing algorithms. RMASE supports a layered architecture, including at least the MAC layer, a routing layer and the application layer, with the MAC layer at the bottom and the application layer at the top. It is the algorithm designer's choice to put individual functions at different layers so that different algorithms can share common functions.

\section{Results and Discussions}

Here, we have used a real application to test the performance of the energy-aware and shortest path protocols. The application, Pursuer Evader Game (PEG) [19], is used for the sensor network to detect an evader and to inform the pursuer about its location. The communication problem in this task is to route packets sent out by one of the sensor nodes to the mobile pursuer. The source is changing from node to node, following the movement of the evader and the destination is mobile. In our tests, the network is a $7 \times 7$ sensor grid with small random offsets. The maximum radio range is about $3 \mathrm{~d}$, where $\mathrm{d}$ is the standard distance between two neighbour nodes in the grid. Figure 2 shows an instance of the connectivity of such a network.

The normal radio model (NRM, default radio model in PROWLER), radio model with SINR (RMSINR), radio model with Rayleigh fading (RMRYF) and radio model developed with Rician fading (RMRCF) are used in our experiments. The radio data rate is $40 \mathrm{kbps}$ [20] for MICA and each packet has 960 bits. On the other hand, for MICAz motes the radio data rate is $250 \mathrm{kbps}$ [21] with each packet having 960 bits. The application sends out one packet per second from the sources. The results are based on the average of 10 random runs. The other parameters used for MICA and MICAz motes are mentioned in Table 1.

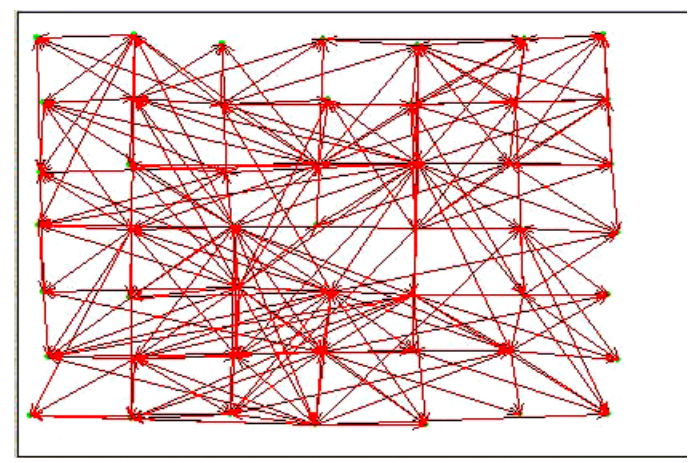

Figure 2. Instance of radio connectivity. 
Figure 3 indicates the comparison of latency with $\mathrm{CF}$, ATS \& AT for different radio models in case of MICA and MICAz. From Figure 3 it has been observed that for realistic model RMRCF the latency is highest that with all routing protocols under consideration while it is minimum in case of NRM. Here the results reveal that the performance AT routing protocol is proved to be vigorous under the influence of all kind of fading radio models in both cases of MICA and MICAz motes. Moreover it has been observed that latency is less in case of MICAz with respect to mica for all types of routing algorithms under consideration.

Table 1. Parameters for MICA and MICAz motes.

\begin{tabular}{lcc}
\hline & MICA & MICAz \\
\hline Chipset & TR 1000 & CC 2420 \\
Radio frequency $[\mathrm{MHz}]$ & $433 / 915$ & 2400 to 2483.5 \\
Max data rate $(\mathrm{kbps})$ & 40 & 250 \\
RX power $(\mathrm{mW})$ & 3.8 & 19.7 \\
TX power $(\mathrm{mW})$ & 12 & 17.4 \\
Powerdown power $(\mu \mathrm{A})$ & 0,7 & 1 \\
Turn on time $(\mathrm{ms})$ & 0.02 & 1.2 \\
Modulation & ASK & DSSS-O-QPSK \\
Receive sensitivity & $-95 \mathrm{dBm}$ & $-94 \mathrm{dBm}$ \\
Outdoor range & To 92 m & $75 \mathrm{~m}$ to $100 \mathrm{~m}$ \\
Multichannel & no & yes \\
\hline
\end{tabular}

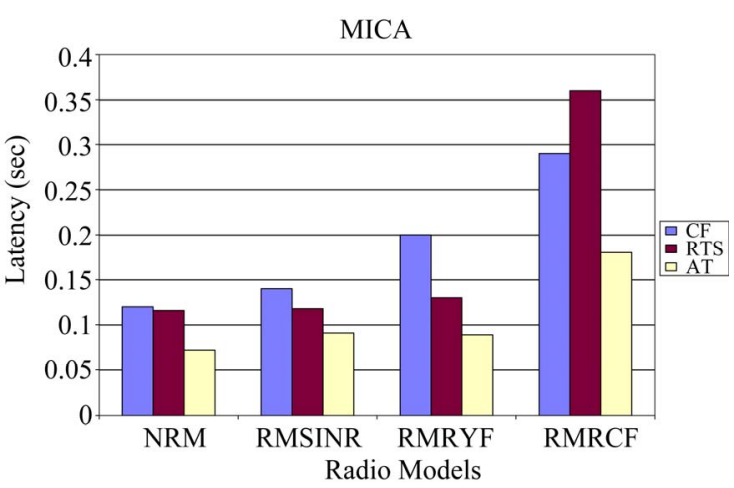

(a)

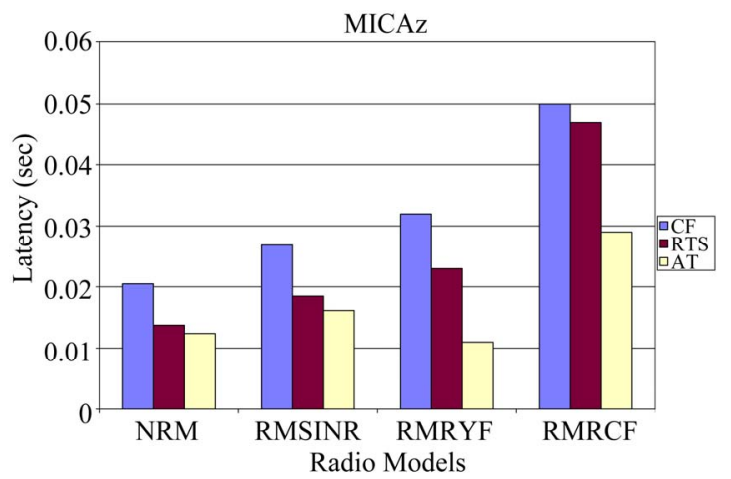

(b)

Figure 3. Latency with CF, ATS \& AT for different radio models in case of (a) MICA (b) MICAz.
Throughput with CF, ATS \& AT for different radio models in case of MICA and MICAz has been shown in Figure 4. The results obtained prove that the throughput highest with $\mathrm{CF}$ routing protocol while it is moderate for AT protocol for all types of radio models under consideration. MICA vis-à-vis MICAz indicates improved throughput for MICAz.

Results for loss rate with CF, ATS \& AT for different radio models in case of MICA and MICAz are shown in Figure 5. From Figure 5 (a) it has been observed that the loss rate negligible in case of RMRYF for CF protocol with mica mote while there is no loss rate for other radio models with both type of motes. Similarly results (Figure 6) have been reported for success rate and CF protocol proved to be the best for both types of motes.

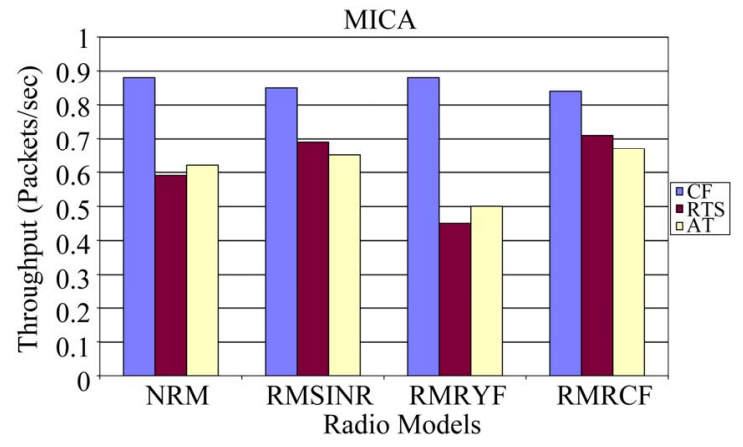

(a)

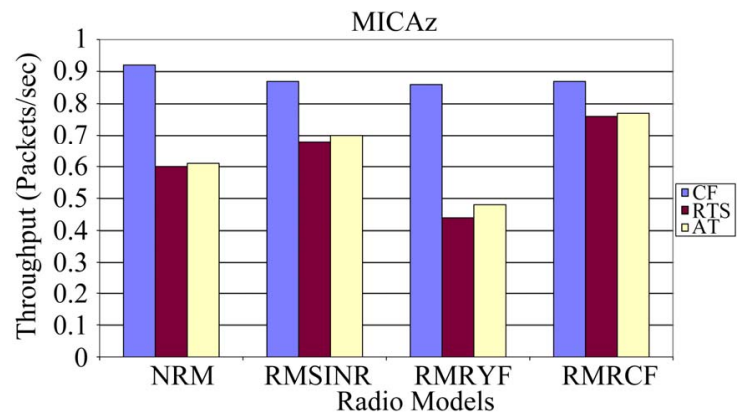

(b)

Figure 4. Throughput with CF, ATS \& AT for different radio models in case of (a) MICA (b) MICAz.

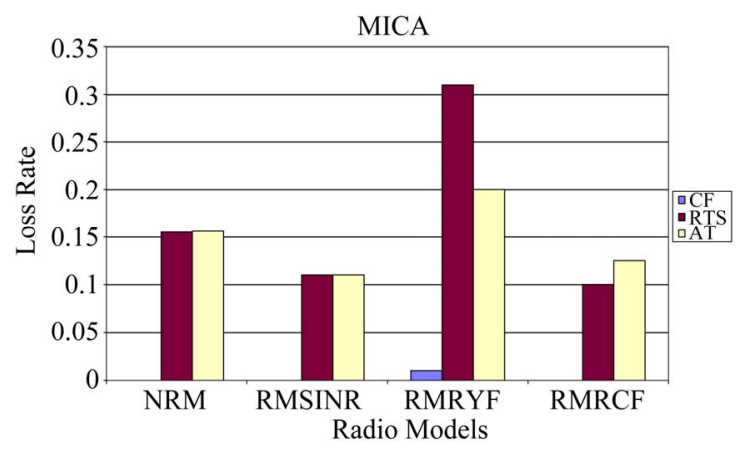

(a) 


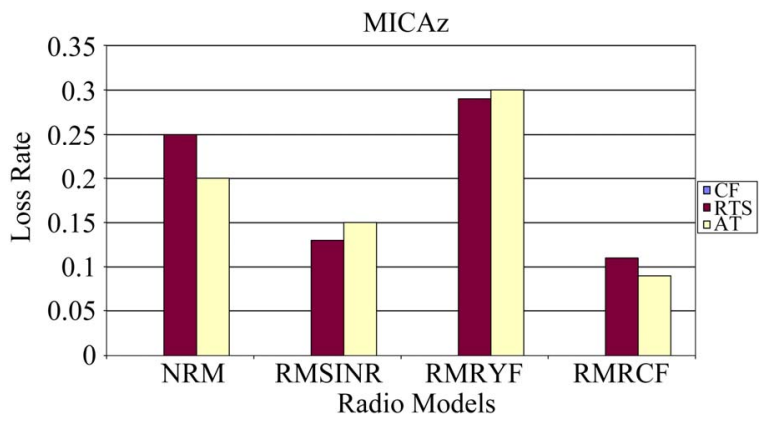

(b)

Figure 5. Loss Rate with CF, ATS \& AT for different radio models in case of (a) MICA (b) MICAz.

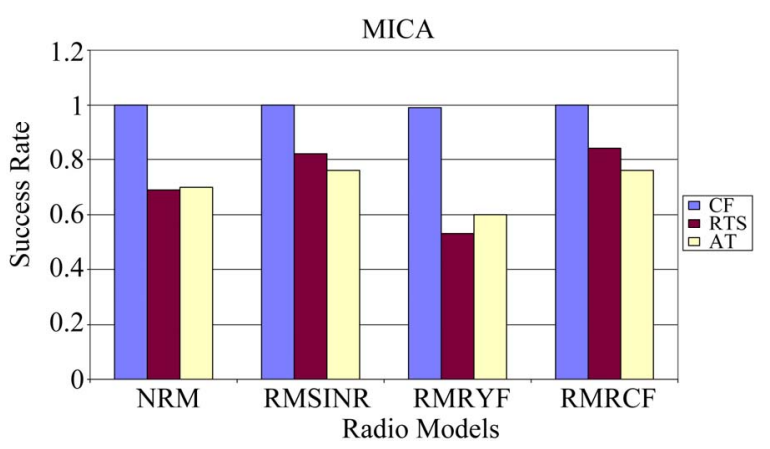

(a)

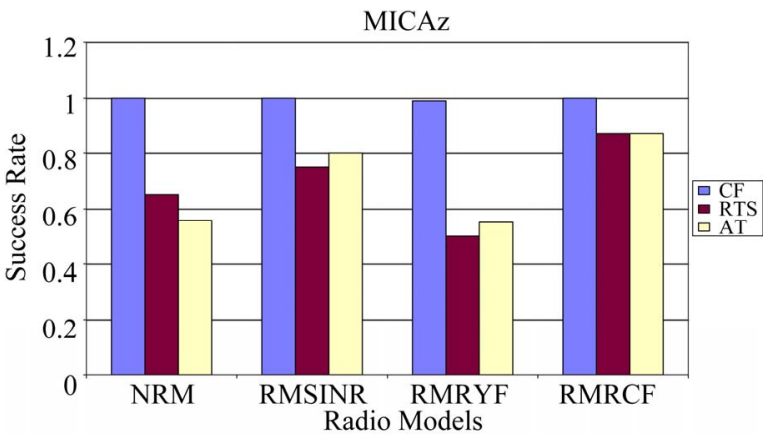

(b)

Figure 6. Success rate with CF, ATS \& AT for different radio models in case of (a) MICA (b) MICAz.

Energy consumption with CF, ATS \& AT for different radio models in case of MICA and MICAz have been shown in Figure 7. Results indicate that the energy consumption is minimum in case of NRM and RMRYF for RTS for MICA and MICAz respectively. On the other hand for realistic radio model RMRCF the energy consumed is minimum for AT in comparison with CF and RTS.

In Figure 8 efficiency results ascertain that the CF routing protocol proved to be feeble with all types of radio models. Figure 9 shows the lifetime with CF, ATS $\&$ AT for different radio models in case of MICA and MICAz. The results obtained establish that for realistic

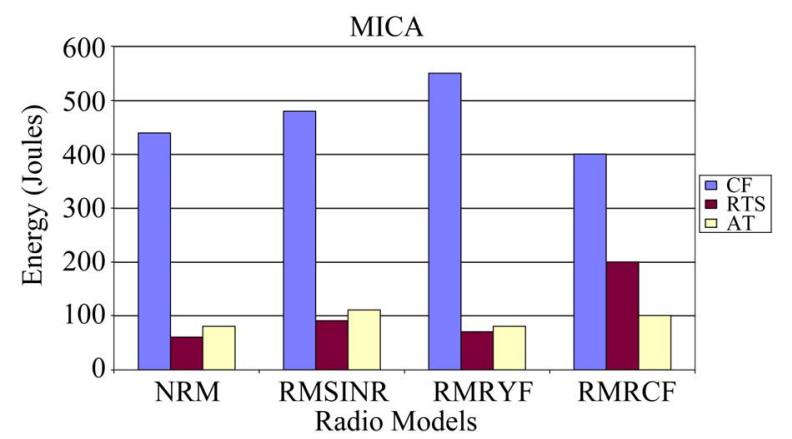

(a)

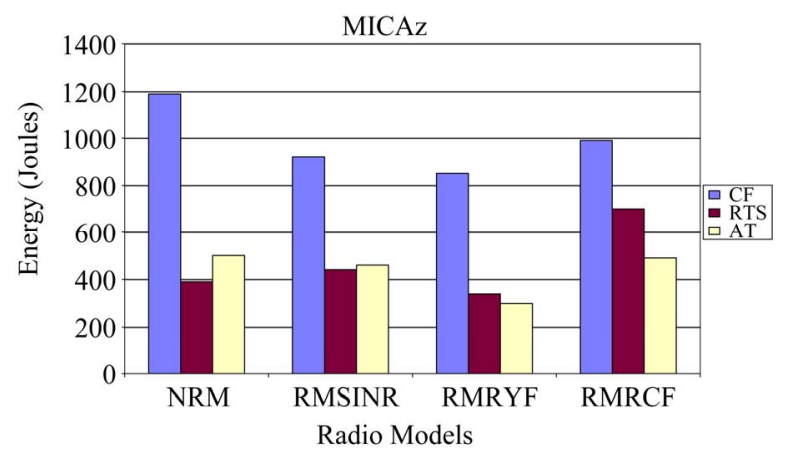

(b)

Figure 7. Energy with CF, ATS \& AT for different radio models in case of (a) MICA (b) MICAz.

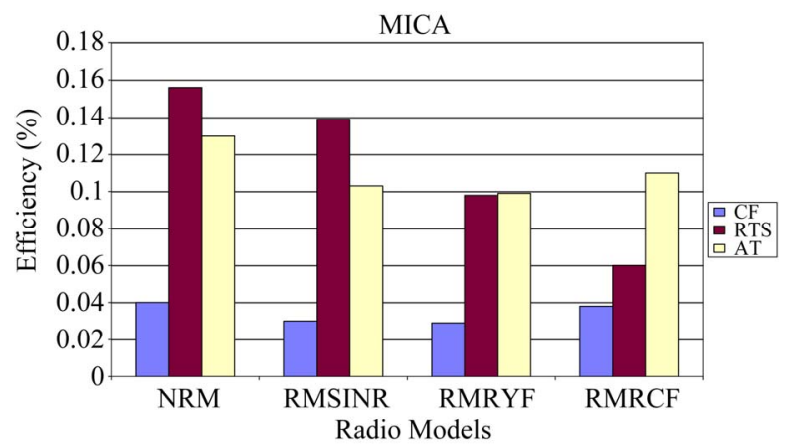

(a)

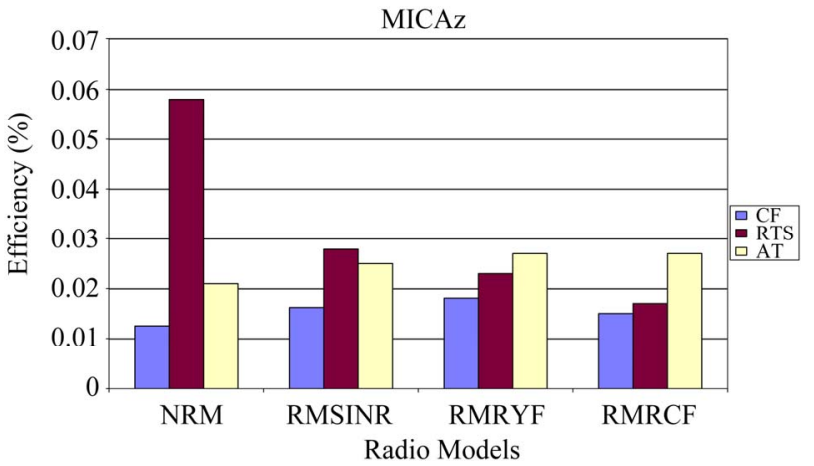

(b)

Figure 8. Efficiency with CF, ATS \& AT for different radio models in case of (a) MICA (b) MICAz. 


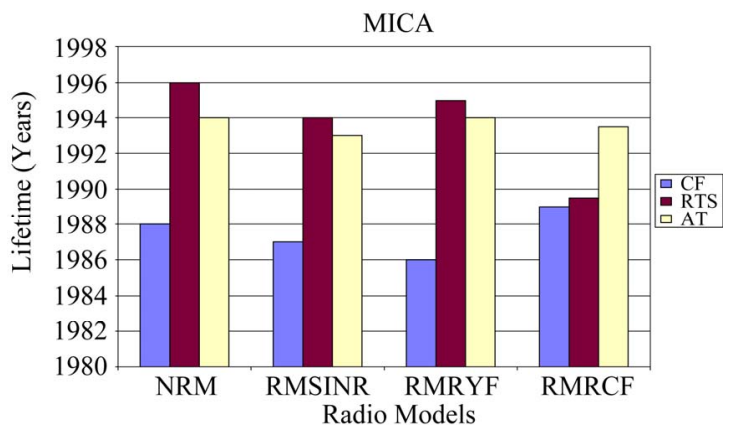

(a)

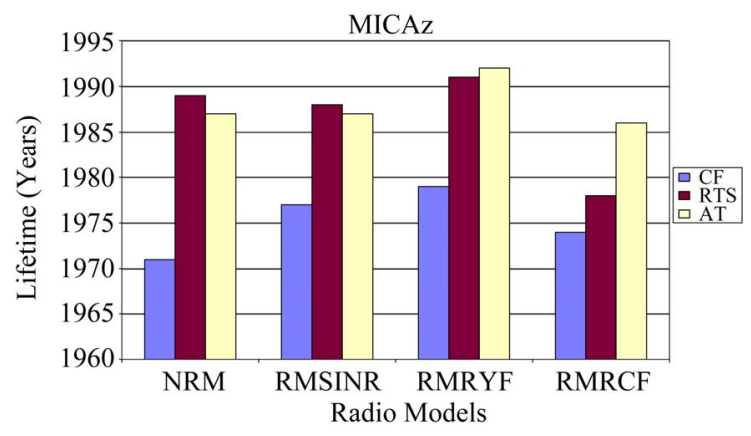

(b)

Figure 9. Lifetime with CF, ATS \& AT for different radio models in case of (a) MICA (b) MICAz.

model RMRCF the lifetime with AT routing protocol is more in comparison with CF and ATS protocols. The results obtained in this paper establish that the behaviour of routing protocols is different with various radio models under consideration. Therefore it evident from discussion that there is performance trade-off with routing protocols for radio models for wireless sensor networks. The results ascertain that the MICAz motes give low latency, high throughput, high energy consumption, low efficiency but better lifetime while the MICA motes give high success rate and less loss rate for all routing protocols but there performance trade-off that is dependent of radio models. It has been, thus, concluded that in case of all the radio models the MICAz is preferably better than MICA in applications where energy is not a constrain. For greater lifetime applications MICAz has to be preferred over MICA for all radio models.

\section{Conclusions}

In this paper, the simulation results on the performance of routing protocols Constrained Flooding (CF), RealTime Search (RTS) and Adaptive Tree (AT) for wireless sensor networks using different radio models have been presented. The investigations have been reported for MICA and MICAz motes and are based on various performance metrics like: latency, loss rate, success rate, throughput, energy consumption, efficiency and lifetime.
It has been observed that the latency in case of MICAz with respect to MICA for all types of routing algorithms under consideration is less. However throughput is highest with $\mathrm{CF}$ routing protocol for all the radio models. On the other hand, loss rate is insignificant in case of RMRYF for CF protocol with MICA mote while it is zero for other radio models with both types of motes. Similarly the success rate proves to be the best in case of $\mathrm{CF}$ protocol for both types of motes. The energy consumption is low in presence of NRM and RMRYF for RTS in case of MICA and MICAz respectively. For realistic radio model RMRCF the energy consumed is found to be minimum for AT protocol in comparison with $\mathrm{CF}$ and RTS. In case of efficiency, the results obtained indicate that the CF routing protocol proves to be weak with all types of radio models. Moreover, the results indicate that for realistic model RMRCF the lifetime with AT routing protocol is significantly high in comparison with $\mathrm{CF}$ and RTS protocols. Finally, it is concluded that the AT protocol can be applied to achieve better energy consumption, efficiency and lifetime in real time for wireless sensor networks.

\section{REFERENCES}

[1] J. N. Al-Karaki and A. E. Kamal, "A Taxonomy of Routing Techniques in Wireless Sensor Networks," In M. Ilyas and I. Mahgoub, Eds., Handbook of Sensor Networks: Compact Wireless and Wired Sensing Systems, Chapter 6, CRC Press, Boca Raton, 2005, pp. 1-24.

[2] B. Karp and H. T. Kung, "GPSR: Greedy Perimeter Stateless Routing for Wireless Networks," Proceedings of 6th International Conference on Mobile Computing and Networks, Boston, 6-11 August 2000, pp. 243-254. doi: $10.1145 / 345910.345953$

[3] M. Chu, H. Haussecker and F. Zhao, "Scalable Information-Driven Sensor Querying and Routing for Ad Hoc Heterogeneous Sensor Networks," International Journal of High Performance Computing Applications, Vol. 16, No. 3, 2002, pp. 293-313. doi:10.1177/10943420020160030901

[4] Y. Yu, R. Govindan and D. Estrin, "Geographical and Energy Aware Routing: A Recursive Data Dissemination Protocol for Wireless Sensor Networks," Technical Report UCLA/CSD-TR-01-0023, Computer Science Department, University of California, Los Angeles, May 2001.

[5] Y. Zhang and M. P. J. Fromherz, "Message-Initiated Constraint-Based Routing for Wireless Ad-Hoc Sensor Networks," Proceedings of 1st IEEE Consumer Communication and Networking Conference, Las Vegas, 5-8 January 2004, pp. 648-650.

[6] Y. Zhang, M. P. J. Fromherz and L. D. Kuhn, "Smart Routing with Learning-Based QoS-Aware Meta-Strategies," Proceedings of 1 st Workshop Quality of Service Routing, Barcelona, 1 October 2004, pp. 298-307.

[7] Y. Zhang and M. Fromherz, "Search-Based Adaptive 
Routing Strategies for Sensor Networks," Proceedings of AAAI Sensor Networks Workshop, San Jose, 26 July 2004, pp. 1-8.

[8] Y. Zhang and M. P. J. Fromherz, "Constrained Flooding: A Robust and Efficient Routing Framework for Wireless Sensor Networks," Proceedings of 20th IEEE International Conference on Advanced Information Networking and Applications, Vienna, 18-20 April 2006, pp. 1-6.

[9] Y. Zhang and Q. F. Huang, "Adaptive Tree: A LearningBased Meta-Routing Strategy for Sensor Networks," Proceedings of 3rd IEEE Consumer Communications and Networking Conference, 8-10 January 2006, pp. 122-126.

[10] Y. Zhang, G. Simon and G. Balogh, "High-Level Sensor Network Simulations for Routing Performance Evaluations," Proceedings of 3rd International Conference on Networked Sensing Systems, Chicago, 31 May-2 June 2006, pp. 1-4.

[11] S. Park, A. Savvides and M. B. Srivastava, "SensorSim: A Simulation Framework for Sensor Networks," Proceedings of the 3rd ACM International Workshop on Modeling, Analysis and Simulation of Wireless and Mobile Systems, Boston, 20 August 2000, pp. 104-111.

[12] P. Levis, N. Lee, M. Welsh and D. Culler, "TOSSIM: Accurate and Scalable Simulation of Entire TinyOS Applications," Proceedings of the 1st International Conference on Embedded Networked Sensor Systems, Los Angeles, 5-7 November 2003, pp. 126-137. doi:10.1145/958491.958506

[13] K. Fall and K. Varadhan, "The VINT Project," The NS Manual, November 2008.

http://www.isi.edu/nsnam/ns/doc/
[14] OPNET Technologies, Inc., "The OPNET Simulator," Bethesda. http://www.opnet.com

[15] G. Simon, "Prowler: Probabilistic Wireless Network Simulator," Institute for Software Integrated Systems, Nashville, 2003. http://www.isis.vanderbilt.edu/projects/nest/prowler

[16] G. Simon, P. Volgyesi, M. Maroti and A. Ledeczi, "Simulation-Based Optimization of Communication Protocols for Large-Scale Wireless Sensor Networks," Proceedings of IEEE International Aerospace Conference, Big Sky, Vol. 3, 8-15 March 2003, pp. 1339-1346.

[17] M. Haenggi, "Probabilistic Analysis of a Simple Mac Scheme for Ad Hoc Wireless Networks," Proceedings of IEEE CAS Workshop on Wireless Communications and Networking, Pasadena, 5-6 September 2002, pp. 1-4.

[18] U. C. Berkeley Wireless Embedded Systems, 2003. http://webs.cs.berkeley.edu

[19] Y. Zhang and M. Fromherz, "A Robust and Efficient Flooding-Based Routing for Wireless Sensor Networks," Journal of Interconnection Networks, Vol. 7, No. 4, 2006, pp. 549-568. doi:10.1142/S0219265906001855

[20] Crossbow: MICA, Wireless Measurement System Datasheet.

http://www.xbow.com/Products/Product_pdf_files/Wirele ss_pdf/MICA.pdf

[21] Crossbow: MICAz, Wireless Measurement System Datasheet.

http://www.xbow.com/Products/Product pdf files/Wirele ss_pdf/MICAz_Datasheet.pdf 